\title{
HEIGHT PAIRINGS FOR ALGEBRAIC CYCLES ON ABELIAN VARIETIES
}

\author{
BY KLAUS KÜNNEMANN
}

\begin{abstract}
Beilinson and Bloch have given conditional constructions of height pairings between algebraic cycles on smooth projective varieties over number fields. These pairings generalize the classical Néron-Tate height pairing between divisors and zero-cycles and give conjecturally a description of the behavior of motivic $L$-functions near the central point. We give an unconditional construction of height pairings for algebraic cycles on abelian varieties. This improves a previous result where we have defined height pairings on abelian varieties which have totally degenerate reduction at all places of bad reduction. Our construction is based on the existence of projective regular models for abelian varieties over number fields and on the study of cycles on degenerate fibers in mixed characteristic initiated by Bloch, Gillet, and Soulé.
\end{abstract}

○ 2001 Éditions scientifiques et médicales Elsevier SAS

RÉSUMÉ. - Beilinson et Bloch ont proposé une définition conjecturale des accouplements de hauteur entre cycles algébriques sur les variétés projectives lisses definies sur un corps de nombres. Ces accouplements généralisent l'accouplement de Néron-Tate entre zéro-cycles et diviseurs. Ils permettent de décrire conjecturalement le comportement des fonctions $L$ motiviques au centre de leurs bandes critiques. Nous proposons une construction inconditionnelle des accouplements de hauteurs entre cycles algébriques sur les variétés abéliennes, qui généralise notre construction antérieure pour les variétés abéliennes à réduction totalement dégénérée. Notre construction repose sur l'existence de modèles projectifs réguliers pour les variétés abéliennes sur les corps de nombres, et d'autre part sur l'étude des cycles sur les fibres dégénérées en caractéristique mixte initiée par Bloch, Gillet et Soulé.

○) 2001 Éditions scientifiques et médicales Elsevier SAS

\section{Introduction}

Let $A_{K}$ be an abelian variety which is defined over a number field $K$. Let $C H^{p}\left(A_{K}\right)^{0}$ be the Chow group of homologically trivial cycles of codimension $p$ on $A_{K}$. The aim of this paper is to show that there is a well defined height pairing

$$
\langle., .\rangle_{A_{K}}: C H^{p}\left(A_{K}\right)^{0} \times C H^{\operatorname{dim} A_{K}+1-p}\left(A_{K}\right)^{0} \longrightarrow \mathbb{R}
$$

which generalizes the classical Néron-Tate height pairing between divisors and zero-cycles. The precise statement of our result is given in Theorem 1.6. This improves a result in [12] where we have constructed the above height pairing under the assumption that the abelian variety $A_{K}$ has totally degenerate reduction at all places of bad reduction. Beilinson and Bloch have given in [1] and [2] conditional definitions of height pairings for homologically trivial cycles on any smooth 
projective variety which is defined over a number field. Conjecturally these height pairings can be used to describe the behavior of motivic $L$-functions near the central point. We use a variant of Beilinson's construction to define the pairing (1) which is based on arithmetic intersection theory as developed by Gillet and Soulé. In order to define a height pairing for algebraic cycles on $A_{K}$, we have to overcome two difficulties. We need a projective regular model $P$ of $A_{K}$ over the ring of integers $\mathcal{O}_{K}$ in $K$ and extensions of homologically trivial cycles on $A_{K}$ to $P$ which are perpendicular to cycles supported in a special fiber with respect to the arithmetic intersection pairing on the model $P$.

Using results of Mumford, Chai, and Faltings, we have constructed in [12] projective regular models for abelian varieties with semi-abelian reduction. We have shown furthermore that an abelian variety $A_{K}$ has potentially semi-stable reduction, i.e., locally at primes of $\mathcal{O}_{K}$ there exists after a finite flat base change a projective strictly semi-stable model $P$ of $A_{K}$. The special fiber of a strictly semi-stable model has a canonical stratification. The strata in the special fiber of the strictly semi-stable models of abelian varieties constructed in [12] have the following description. Each stratum is a semi-abelian scheme $G_{\alpha}$ which is an extension of an abelian variety $A_{\alpha}$ by a split torus $T_{\alpha}$. The closure $P_{\alpha}$ of $G_{\alpha}$ in $P$ is a contraction product

$$
P_{\alpha}=G_{\alpha} \times{ }^{T_{\alpha}} Z_{\alpha}
$$

for some smooth projective toric variety $T_{\alpha} \hookrightarrow Z_{\alpha}$.

The problem to find suitable extensions of homologically trivial cycles on $A_{K}$ to a projective regular model can be checked locally at primes of $\mathcal{O}_{K}$. In [12], we have investigated this question for strictly semi-stable models. Let $S$ be the spectrum of a discrete valuation ring with finite residue class field $k$. Let $P$ be a projective strictly semi-stable $S$-model of a $d$-dimensional abelian variety $A_{K}$ as described above. We denote by $i: Y \hookrightarrow P$ the special fiber of $P$ and by $Y^{(r)}$ the disjoint union of the $r$-fold intersections of the irreducible components of $Y$. The natural inclusions can be used to define canonical maps

$$
\gamma: A^{d-p-1}\left(Y^{(2)}\right) \rightarrow A^{d-p}\left(Y^{(1)}\right), \quad \rho: A^{p}\left(Y^{(1)}\right) \rightarrow A^{p}\left(Y^{(2)}\right)
$$

between the groups $A^{\cdot}\left(Y^{(r)}\right)$ of algebraic cycles on $Y^{(r)}$ modulo homological equivalence. We define $A_{p}(Y)=\operatorname{Coker}(\gamma)$ and $A^{p}(Y)=\operatorname{ker}(\rho)$. We are looking for extensions of homologically trivial cycles on the generic fiber of $A_{K}$ to $P$ which are perpendicular to cycles supported on $Y$ with respect to the local arithmetic intersection pairing. This problem has a solution if the cycle class map induces an injection

$$
\mathrm{cl}: A_{p}(Y) \longrightarrow H_{2 p}^{\text {ét }}\left(Y \otimes_{k} \bar{k}, \mathbb{Q}_{l}(-p)\right)
$$

and the complex

$$
A_{d+1-p}(Y) \stackrel{i^{*} i_{*}}{\longrightarrow} A^{p}(Y) \stackrel{\cdot \cap[Y]}{\longrightarrow} A_{d-p}(Y)
$$

is exact. Using results about cycles on degenerate fibers in mixed characteristic of Bloch, Gillet, and Soulé [3], we have seen in [12] that (3) is injective and (4) is exact if the varieties $P_{\alpha}$ satisfy Grothendieck's standard conjectures and the Tate conjecture. In the special case where $A_{K}$ has totally degenerate reduction at all places of bad reduction, each $P_{\alpha}$ is a smooth projective toric variety and satisfies Grothendieck's standard conjectures and the Tate conjecture. This is sufficient to show in [12] that there is a well defined height pairing on $A_{K}$. For general abelian varieties, arbitrary contraction products (2) may appear as closures of strata in a model. We have shown in [14] that Grothendieck's standard conjectures and the Tate conjecture hold for a 
contraction product (2) if they hold for the abelian variety $A_{\alpha}$. However we do not know that these conjectures hold for arbitrary abelian varieties. Therefore we use a different argument for general abelian varieties. The ring of algebraic cycles modulo homological equivalence on a contraction product (2) can be computed as

$$
A^{\prime}\left(P_{\alpha}\right)=A^{\cdot}\left(A_{\alpha}\right) \otimes_{\mathbb{Q}} A^{\cdot}\left(Z_{\alpha}\right) .
$$

This isomorphism allows us to express the injectivity in (3) and the exactness of (4) in terms of corresponding statements involving only the toric varieties $Z_{\alpha}$. We reduce our problem to the case of totally degenerate reduction in the following way. We use Mumford's construction to produce a second abelian variety $A_{K}^{\prime}$ with totally degenerate reduction together with a model $P^{\prime}$ which is related to the original model $P$ as follows. The closures of the strata in the special fiber of $P^{\prime}$ are precisely the toric varieties $Z_{\alpha}$ which appear in the contraction products $P_{\alpha}$ of the original model. Furthermore the simplicial complex which describes how the different strata are related to each other coincides for $P$ and $P^{\prime}$. This allows us to conclude the injectivity in (3) and the exactness of (4) from the corresponding statements for $P^{\prime}$.

\section{Notations and conventions}

For an abelian group $X$, we define $X_{\mathbb{Q}}=X \otimes_{\mathbb{Z}} \mathbb{Q}, X_{\mathbb{R}}=X \otimes_{\mathbb{Z}} \mathbb{R}$, and $X^{*}=\operatorname{Hom}_{\mathbb{Z}}(X, \mathbb{Z})$. The dual abelian variety of an abelian variety $A$ is denoted by $A^{t}$. Let $S$ denote the spectrum of a field or a Dedekind domain. Let $X$ be a separated $S$-scheme of finite type. We denote by $\mathrm{CH}_{p}(\mathrm{X})$ the Chow group of algebraic cycles of dimension $p$ on $X$ modulo rational equivalence as defined in [7, 20.1]. However we take $p$ to be the absolute dimension over $S$, which is the relative dimension over $S$ plus the dimension of $S$. If $X$ is regular and equidimensional of finite Krull dimension $d$, we write $C H^{p}(X)=C H_{d-p}(X)$.

\section{Height pairings for algebraic cycles}

1.1. Let $K$ be a number field, $\mathcal{O}_{K}$ its ring of integers, and $X_{\eta}$ a smooth projective variety of dimension $d$ over $\eta=\operatorname{Spec} K$. The Chow group of homologically trivial cycles on $X_{\eta}$ is the kernel of the cycle class map

$$
C H^{p}\left(X_{\eta}\right)_{\mathbb{Q}}^{0}=\operatorname{ker}\left(\mathrm{cl}: C H^{p}\left(X_{\eta}\right)_{\mathbb{Q}} \longrightarrow H_{\text {ét }}^{2 p}\left(X_{\bar{\eta}}, \mathbb{Q}_{l}(p)\right)\right) .
$$

Bloch and Beilinson have given in [1], [2] conditional definitions of height pairings

$$
\langle., .\rangle_{X_{\eta}}: C H^{p}\left(X_{\eta}\right)_{\mathbb{Q}}^{0} \times C H^{d+1-p}\left(X_{\eta}\right)_{\mathbb{Q}}^{0} \longrightarrow \mathbb{R}
$$

We recall a definition of Beilinson's pairing which requires the following assumptions:

1.2. Assumption. - The variety $X_{\eta}$ has a regular model $X$ which is flat and projective over $S=\operatorname{Spec} \mathcal{O}_{K}$

Once we have a regular model, we can use arithmetic intersection theory [10, 4.3.8] to define a pairing

$$
\langle., .\rangle_{X}: C H^{p}(X)_{\mathbb{Q}}^{0} \times C H^{d+1-p}(X)_{\mathbb{Q}}^{0} \longrightarrow \mathbb{R}
$$


where $C H^{p}(X)_{\mathbb{Q}}^{0}$ denotes the subspace of $C H^{p}(X)_{\mathbb{Q}}$ consisting of cycle classes whose restriction to $X_{\eta}$ is homologically trivial. Let

$$
C H_{\mathrm{fin}}^{p}(X)_{\mathbb{Q}}=\operatorname{ker}\left(C H^{p}(X)_{\mathbb{Q}} \longrightarrow C H^{p}\left(X_{\eta}\right)_{\mathbb{Q}}\right) .
$$

We define $C H_{\text {fin }}^{p}(X)_{\mathbb{Q}}^{\perp}$ to be the orthogonal complement to $C H_{\text {fin }}^{p}(X)_{\mathbb{Q}}$ with respect to the pairing (6). There is a canonical map

$$
\varphi_{X}: C H_{\text {fin }}^{d+1-p}(X) \stackrel{\perp}{\mathbb{Q}} \longrightarrow C H^{p}\left(X_{\eta}\right)_{\mathbb{Q}}^{0} .
$$

1.3. Assumption. - The map $\varphi_{X}$ is surjective.

If the Assumptions 1.2 and 1.3 hold, we can define the pairing (5) by applying the pairing (6) to preimages under $\varphi_{X}$. We obtain height pairings (5) which behave well under the action of correspondences and generalize the classical Néron-Tate height pairing between divisors and zero-cycles [1], [2], [11].

1.4. Let $K^{\prime}$ be a finite extension of $K, X_{\eta}^{\prime}=X_{\eta} \otimes_{K} K^{\prime}$, and denote by $q$ the natural map from $X_{\eta}^{\prime}$ to $X_{\eta}$. If our Assumptions 1.2 and 1.3 hold for $X_{\eta}$ and $X_{\eta}^{\prime}$ then it follows from Lemma 1.5 below that

$$
\langle\alpha, \beta\rangle_{X_{\eta}}=\frac{1}{\left[K^{\prime}: K\right]}\left\langle q^{*} \alpha, q^{*} \beta\right\rangle_{X_{\eta}^{\prime}}
$$

holds for all $\alpha, \beta \in C H^{\cdot}\left(X_{\eta}\right)_{\mathbb{Q}}^{0}$. We use Eq. (7) to define the pairing (5) if 1.2 and 1.3 hold for $X_{\eta}^{\prime}$ for some finite extension $K^{\prime}$ of $K$. The following lemma shows that the definition of (5) via (7) is independent of choices. It doesn't depend on the choice of a field $K^{\prime}$ and on the choice of a model over $\mathcal{O}_{K^{\prime}}$ satisfying our Assumptions 1.2 and 1.3.

1.5. Lemma. - Let $K_{i}, i=1,2$, be finite extensions of $K$ and $\eta_{i}=\operatorname{Spec} K_{i}$. We assume that $X_{\eta_{i}}=X_{\eta} \otimes_{\eta} \eta_{i}$ admits a model $X_{i}$ over $\mathcal{O}_{K_{i}}$ satisfying 1.2 and 1.3. Let $q_{i}$ denote the natural map from $X_{\eta_{i}}$ to $X_{\eta}$. We have

$$
\frac{1}{\left[K_{1}: K\right]}\left\langle q_{1}^{*} \alpha, q_{1}^{*} \beta\right\rangle_{X_{\eta_{1}}}=\frac{1}{\left[K_{2}: K\right]}\left\langle q_{2}^{*} \alpha, q_{2}^{*} \beta\right\rangle_{X_{\eta_{2}}}
$$

for all $\alpha \in C H^{p}\left(X_{\eta}\right)_{\mathbb{Q}}^{0}$ and $\beta \in C H^{d+1-p}\left(X_{\eta}\right)_{\mathbb{Q}}^{0}$.

Proof. - Let $L$ be a finite extension of $K$ which contains $K_{1}$ and $K_{2}$. Let $p_{i}$ denote the natural map from $X_{L}=X_{\eta} \otimes_{K} L$ to $X_{\eta_{i}}$. The variety $X_{L}$ together with $p_{1}$ and $p_{2}$ induces a correspondence $\Gamma_{\eta}=\left(p_{1}, p_{2}\right)_{*}\left[X_{L}\right]$ in $C H^{*}\left(X_{\eta_{1}} \times_{\eta} X_{\eta_{2}}\right)$. The correspondence $\Gamma_{\eta}$ and its transpose $\Gamma_{\eta}^{t}$ induce maps $\Gamma_{\eta, C H}$ and $\Gamma_{\eta, C H}^{t}$ on Chow groups which satisfy

$$
\Gamma_{\eta, C H}\left(q_{1}^{*} \alpha\right)=\left[L: K_{2}\right] q_{2}^{*} \alpha, \quad \Gamma_{\eta, C H}^{t}\left(q_{2}^{*} \alpha\right)=\left[L: K_{1}\right] q_{1}^{*} \alpha
$$

for all $\alpha \in C H^{\cdot}\left(X_{\eta}\right)$. The product $X_{1} \times_{S} X_{2}$ is a model of $X_{\eta_{1}} \times_{\eta} X_{\eta_{2}}$. Let $\Gamma$ be a class in $C H .\left(X_{1} \times{ }_{S} X_{2}\right)$ which restricts to $\Gamma_{\eta}$. For $\gamma \in C H^{p}\left(X_{\eta_{i}}\right)_{\mathbb{Q}}^{0}$, we denote by $\widetilde{\gamma}$ a lift in $C H^{d+1-p}\left(X_{i}\right) \stackrel{\perp}{\mathbb{Q}}$ under $\varphi_{X_{i}}$. By definition and (8), we get

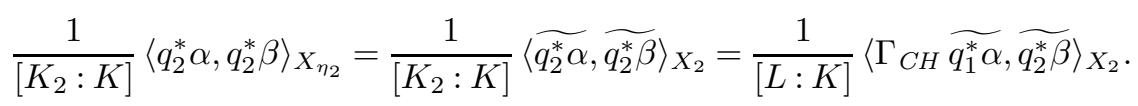


Using the projection formula [11, Lemma 4.1], this equals

$$
\frac{1}{[L: K]}\left\langle\widetilde{q_{1}^{*} \alpha}, \Gamma_{C H}^{t} \widetilde{q_{2}^{*} \beta}\right\rangle_{X_{1}}=\frac{1}{\left[K_{1}: K\right]}\left\langle\widetilde{q_{1}^{*} \alpha}, \widetilde{q_{1}^{*} \beta}\right\rangle_{X_{1}}=\frac{1}{\left[K_{1}: K\right]}\left\langle q_{1}^{*} \alpha, q_{1}^{*} \beta\right\rangle_{X_{\eta_{1}}} .
$$

This proves our claim.

The following theorem is the main result of this paper.

1.6. THEOREM. - Let $A_{\eta}$ be an abelian variety with semi-abelian reduction over $\eta$. Then $A_{\eta}$ admits a regular model $P$ which is flat and projective over $S=\operatorname{Spec}\left(\mathcal{O}_{K}\right)$ for which the map

$$
\varphi_{P}: C H_{\mathrm{fin}}^{\operatorname{dim}} A_{\eta}+1-p(P)_{\mathbb{Q}}^{\perp} \longrightarrow C H^{p}\left(A_{\eta}\right)_{\mathbb{Q}}^{0}
$$

is surjective for all $p \geqslant 0$.

The proof of the theorem will be given in Sections 5 and 6 . Let $A_{\eta}$ be an abelian variety over $\eta$. By the semi-abelian reduction theorem [5, I 2.6], we may assume that $A_{\eta}$ has semiabelian reduction if we replace $K$ by some finite field extension. We conclude from 1.4 and from Theorem 1.6:

\subsection{COROLLARY. - There is a canonical well defined height pairing}

$$
\langle., .\rangle_{A_{\eta}}: C H^{p}\left(A_{\eta}\right)_{\mathbb{Q}}^{0} \times C H^{\operatorname{dim} A_{\eta}+1-p}\left(A_{\eta}\right)_{\mathbb{Q}}^{0} \longrightarrow \mathbb{R}
$$

for every abelian variety $A_{\eta}$ which is defined over a number field $K$.

\section{Semi-stable reduction}

2.1. Let $R$ be a discrete valuation ring with quotient field $K$ and perfect residue class field $k$. We set $S=\operatorname{Spec} R, \eta=\operatorname{Spec} K, S_{0}=\operatorname{Spec} k$ and $X_{0}=X \times{ }_{S} S_{0}$ for an $S$-scheme $X$. Let $X$ be a projective flat $S$-scheme with reduced special fiber $Y=X_{0}$. Let $Y_{1}, \ldots, Y_{t}$ be the irreducible components of $Y$. For $I \subset\{1, \ldots, t\}$, we denote by $Y_{I}$ the scheme-theoretic intersection $\bigcap_{i \in I} Y_{i}$. We have in particular $Y_{\emptyset}=X$. Let $X$ be a projective flat $S$-scheme of pure dimension $d+1$ which is strictly semi-stable over $S$, i.e., the generic fiber $X_{\eta}=X \backslash Y$ of $X$ is smooth over $\eta$, the special fiber $Y=X_{0}$ is reduced, each $Y_{i}$ is an effective Cartier divisor on $X$, and the schemes $Y_{I}$ are regular and have pure codimension $|I|$ in $X$ for each subset $I \subset\{1, \ldots, t\}$.

2.2. We recall some well known constructions from [3]. For every inclusion of subsets $I \subseteq J \subseteq\{1, \ldots, t\}$, we have a closed immersion $u=u_{J I}: Y_{J} \hookrightarrow Y_{I}$ which induces push-forward and pull-back maps

$$
u_{*}: C H^{p}\left(Y_{J}\right) \longrightarrow C H^{p+|J|-|I|}\left(Y_{I}\right)
$$

and

$$
u^{*}: C H^{p}\left(Y_{I}\right) \longrightarrow C H^{p}\left(Y_{J}\right)
$$

on Chow groups. For $r \geqslant 0$, we denote by $Y^{(r)}$ the disjoint union of all $Y_{I}$ with $|I|=r$. For $I=\left\{i_{1}<\cdots<i_{r+1}\right\}$ and $k \in\{1, \ldots, r+1\}$, we set $I_{k}=I \backslash\left\{i_{k}\right\}$. There are unique homomorphisms $\delta_{k *}$ from $C H^{p}\left(Y^{(r+1)}\right)$ to $C H^{p+1}\left(Y^{(r)}\right)$ and $\delta_{k}^{*}$ from $C H^{p}\left(Y^{(r)}\right)$ to 
$C H^{p}\left(Y^{(r+1)}\right.$ such that the restriction of $\delta_{k *}$ to $C H^{p}\left(Y_{I}\right)$ is $\left(u_{I I_{k}}\right)_{*}$ and the component of $\delta_{k}^{*}$ in $C H^{p}\left(Y_{I}\right)$ is $\left(u_{I I_{k}}\right)^{*}$. We define

$$
\begin{gathered}
\gamma: C H^{p}\left(Y^{(r+1)}\right) \rightarrow C H^{p+1}\left(Y^{(r)}\right), \quad \gamma=\sum_{k=1}^{r+1}(-1)^{k-1} \delta_{k *}, \\
\rho: C H^{p}\left(Y^{(r)}\right) \rightarrow C H^{p}\left(Y^{(r+1)}\right), \quad \rho=\sum_{k=1}^{r+1}(-1)^{k-1} \delta_{k}^{*} .
\end{gathered}
$$

These maps satisfy

$$
\rho^{2}=\gamma \circ \rho+\rho \circ \gamma=\gamma^{2}=0
$$

on $C H^{p}\left(Y^{(r)}\right)$ for all $p, r \geqslant 0$ [3, Proof of Lemma 1]. Let $\pi: Y^{(1)} \rightarrow Y$ denote the natural map. We have exact sequences $[12,5.10]$

$$
\begin{gathered}
C H^{d-p-1}\left(Y^{(2)}\right) \stackrel{\gamma}{\longrightarrow} C H^{d-p}\left(Y^{(1)}\right) \stackrel{\pi_{*}}{\longrightarrow} C H_{p}(Y) \longrightarrow 0, \\
0 \longrightarrow C H^{p}(Y) \stackrel{\pi^{*}}{\longrightarrow} C H^{p}\left(Y^{(1)}\right) \stackrel{\rho}{\longrightarrow} C H^{p}\left(Y^{(2)}\right),
\end{gathered}
$$

where $C H^{p}(Y)$ denotes Chow cohomology of $Y$ in the sense of Fulton [7, 17.3]. There is a natural cap product

$$
C H^{p}(Y) \times C H_{q}(Y) \longrightarrow C H_{q-p}(Y), \quad(\alpha, \beta) \mapsto \alpha \cap \beta .
$$

The projection formula in $[7,17.3]$ applied to $\pi$ allows to describe this product in terms of the intersection product on the smooth projective varieties $Y^{(1)}$. We have

$$
\alpha \cap \pi_{*} \beta=\pi_{*}\left(\pi^{*} \alpha \cdot \beta\right) \quad \forall \alpha \in C H^{p}(Y), \beta \in C H^{d-q}\left(Y^{(1)}\right) .
$$

2.3. We fix an algebraic closure $\bar{k}$ of $k$ and a prime $l$ which is invertible on $S$. We denote by $\bar{V}$ the base change of a $k$-scheme $V$ to $\bar{k}$. For $I \neq \emptyset$, we define

$$
A^{p}\left(Y_{I}\right)=\operatorname{Im}\left(\mathrm{cl}: C H^{p}\left(Y_{I}\right)_{\mathbb{Q}} \longrightarrow H_{\text {ét }}^{2 p}\left(\overline{Y_{I}}, \mathbb{Q}_{l}(p)\right) .\right.
$$

The intersection product on $C H^{\cdot}\left(Y_{I}\right)$ induces a ring structure on $A^{\cdot}\left(Y_{I}\right)$. For $\emptyset \neq I \subseteq J \subseteq$ $\{1, \ldots, r\}$, the maps (9) and (10) induce push-forward and pull-back maps

$$
u_{*}: A^{p}\left(Y_{J}\right) \longrightarrow A^{p+|J|-|I|}\left(Y_{I}\right)
$$

and

$$
u^{*}: A^{p}\left(Y_{I}\right) \longrightarrow A^{p}\left(Y_{J}\right) .
$$

2.4. We are going to extend the definitions in 2.3 to the case $I=\emptyset$. The canonical morphism $j: Y^{(1)} \rightarrow X$ yields a push-forward map

$$
j_{*}=\gamma: C H^{\cdot}\left(Y^{(1)}\right)_{\mathbb{Q}} \longrightarrow C H^{\cdot+1}(X)_{\mathbb{Q}} .
$$

$4^{\text {e }}$ SÉRIE - TOME $34-2001-\mathrm{N}^{\circ} 4$ 
There is a natural ring structure on $C H^{\cdot}(X)_{\mathbb{Q}}[9,8.3]$, [12, 5.2]. It follows from [12, 5.3] and (15) that this product satisfies

$$
j_{*}(\alpha) \cdot \beta=j_{*}\left(\alpha \cdot j^{*} \beta\right), \quad \forall \alpha \in C H^{\cdot}\left(Y^{(1)}\right)_{\mathbb{Q}}, \beta \in C H^{\cdot}(X)_{\mathbb{Q}} .
$$

This projection formula shows that the image of

$$
C H^{\cdot}\left(Y^{(1)}\right)_{\mathbb{Q}}^{0}=\operatorname{ker}\left(\mathrm{cl}: C H^{\cdot}\left(Y^{(1)}\right)_{\mathbb{Q}} \longrightarrow H_{\text {ét }}^{2 \cdot}\left(\overline{Y^{(1)}}, \mathbb{Q}_{l}(\cdot)\right)\right.
$$

under (18) defines an ideal $\mathfrak{a}$ in $C H^{\cdot}(X)_{\mathbb{Q}}$. We let $A^{\cdot}(X)=A^{\cdot}\left(Y_{\emptyset}\right)$ denote the quotient of $C H^{\cdot}(X)_{\mathbb{Q}}$ by $\mathfrak{a}$. Using this definition, we can define the map (16) also in the case $I=\emptyset$. The following lemma shows that (17) is defined for $I=\emptyset$ as well.

2.5. LemmA. - We have $j^{*} j_{*} C H^{p}\left(Y^{(1)}\right)_{\mathbb{Q}}^{0} \subseteq C H^{p+1}\left(Y^{(1)}\right)_{\mathbb{Q}}^{0}$.

Proof. - It follows from (13) that we have a commutative diagram

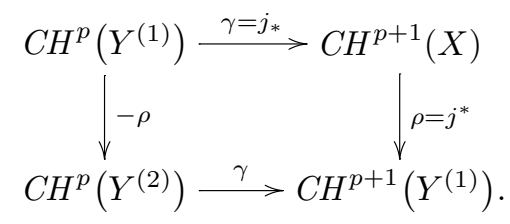

The map $\rho$ on the left-hand side and the map $\gamma$ in the lower arrow are defined in terms of maps (9) and (10) for $I \neq \emptyset$. Hence these maps factor through the groups $A^{\cdot}\left(Y^{(r)}\right)$. This yields our claim.

2.6. The groups $A^{\cdot}\left(Y_{I}\right)$ and the corresponding maps (16) and (17) satisfy functoriality, base change, a projection formula and vanishing of the fundamental class of $Y$ as explained in [3, $1.2 \mathrm{~A} 1-\mathrm{A} 4]$. This holds as the corresponding properties are already satisfied for Chow groups (loc. cit. 1.3.2). The degree of zero cycles defines natural trace maps $\operatorname{tr}_{I}: A^{p}\left(Y_{I}\right) \rightarrow \mathbb{Q}$ for $I \neq \emptyset$ which are compatible with (16). The maps (11) and (12) induce maps

$$
\gamma: A^{p}\left(Y^{(r+1)}\right) \rightarrow A^{p+1}\left(Y^{(r)}\right), \quad \rho: A^{p}\left(Y^{(r)}\right) \rightarrow A^{p}\left(Y^{(r+1)}\right) .
$$

We define

$$
\begin{gathered}
A_{p}(Y)=\operatorname{coker}\left(\gamma: A^{d-p-1}\left(Y^{(2)}\right) \longrightarrow A^{d-p}\left(Y^{(1)}\right)\right), \\
A^{p}(Y)=\operatorname{ker}\left(\rho: A^{p}\left(Y^{(1)}\right) \longrightarrow A^{p}\left(Y^{(2)}\right)\right) .
\end{gathered}
$$

We obtain natural maps from $C H_{p}(Y)$ to $A_{p}(Y)$ and from $C H^{p}(Y)$ to $A^{p}(Y)$. The map from $C H_{p}(Y)_{\mathbb{Q}}$ to $A_{p}(Y)$ is a surjection. The fact that the pairing (14) can be computed on $Y^{(1)}$ implies that there is a corresponding pairing between $A^{p}(Y)$ and $A_{q}(Y)$ with values in $A_{q-p}(Y)$. The maps $\gamma=j_{*}: A^{p-1}\left(Y^{(1)}\right) \rightarrow A^{p}(X)$ and $\rho=j^{*}: A^{p}(X) \rightarrow A^{p}\left(Y^{(1)}\right)$ induce maps

$$
A_{d+1-p}(Y) \stackrel{i_{*}}{\longrightarrow} A^{p}(X) \stackrel{i^{*}}{\longrightarrow} A^{p}(Y) .
$$

The degree map from $\mathrm{CH}_{0}(Y)_{\mathbb{Q}}$ to $\mathbb{Q}$ factors through $A_{0}(Y)$. We consider the following sequence where the second map is induced by the identity on $A^{p}\left(Y^{(1)}\right)$ :

$$
A_{d+1-p}(Y) \stackrel{i^{*} i_{*}}{\longrightarrow} A^{p}(Y) \stackrel{\cap[Y]}{\longrightarrow} A_{d-p}(Y)
$$


It follows from (20) that (22) is a complex.

2.7. LEMMA. - The exactness of the complex (22) is equivalent to the equality

$$
\operatorname{ker}\left(\left.\rho\right|_{A^{p}\left(Y^{(1)}\right)}\right) \cap \operatorname{Im}\left(\left.\gamma\right|_{A^{p-1}\left(Y^{(2)}\right)}\right)=\operatorname{Im}\left(\left.\gamma \circ \rho\right|_{A^{p-1}\left(Y^{(1)}\right)}\right) .
$$

Proof. - This follows from the definitions if one observes $\gamma \circ \rho=-\rho \circ \gamma$.

We observe that the equality in the lemma involves only the strata of $Y$ and their natural inclusion maps. It does not refer to the whole model $X$.

2.8. It is shown in [3, Theorem 5] (compare also [13, 2.15]) that the complex (22) is exact if the $\mathbb{Q}$-vector spaces $A^{\cdot}\left(Y_{I}\right)$ are finite dimensional and satisfy a hard Lefschetz and a Hodge index theorem.

2.9. In the following, we assume that the residue class field $k$ of $R$ is finite. According to Deligne [4, 3.3.4], the cohomology groups $H^{p}=H_{\text {ét }}^{2 p}\left(\bar{Y}, \mathbb{Q}_{l}(p)\right)$ carry a weight filtration $W \cdot H^{p}=W \cdot H_{\text {ét }}^{2 p}\left(\bar{Y}, \mathbb{Q}_{l}(p)\right)$ with weights $\leqslant 0$. The étale homology groups $H_{p}=$ $H_{2 p}^{\text {ét }}\left(\bar{Y}, \mathbb{Q}_{l}(-p)\right)$ are dual to étale cohomology and carry a weight filtration $W . H_{p}=$ $W . H_{2 p}^{\text {ét }}\left(\bar{Y}, \mathbb{Q}_{l}(p)\right)$ with weights $\geqslant 0$. We have

$$
H_{2 p}^{\text {ét }}\left(\bar{Y}, \mathbb{Q}_{l}(-p)\right)^{\operatorname{Gal}(\bar{k} / k)} \subseteq W_{0} H_{2 p}^{\text {ét }}\left(\bar{Y}, \mathbb{Q}_{l}(-p)\right)=\operatorname{Gr}_{0}^{W} H_{2 p}^{\text {ét }}\left(\bar{Y}, \mathbb{Q}_{l}(-p)\right) .
$$

We have derived in $[12,5.11]$ from the spectral sequence of cohomological descent that the cokernel of the map

$$
H_{\text {ét }}^{2 d-2 p-2}\left(\overline{Y^{(2)}}, \mathbb{Q}_{l}(d-p-1)\right) \stackrel{\gamma}{\longrightarrow} H_{\text {ét }}^{2 d-2 p}\left(\overline{Y^{(1)}}, \mathbb{Q}_{l}(d-p)\right)
$$

is $\operatorname{Gr}_{0}^{W} H_{2 p}^{\text {ét }}\left(\bar{Y}, \mathbb{Q}_{l}(-p)\right)$. The map (24) is via the cycle class map compatible with the corresponding map in (21). Hence there is a natural induced map

$$
A_{p}(Y) \longrightarrow \operatorname{Gr}_{0}^{W} H_{2 p}^{\text {ét }}\left(\bar{Y}, \mathbb{Q}_{l}(-p)\right)
$$

which we denote by $\mathrm{cl}$ as well. It follows from the functorial behavior of the cycle class map in étale homology that it factors as

$$
\mathrm{cl}: C H_{p}(Y) \longrightarrow A_{p}(Y) \longrightarrow \operatorname{Gr}_{0}^{W} H_{2 p}^{\text {ét }}\left(\bar{Y}, \mathbb{Q}_{l}(-p)\right) \subseteq H_{2 p}^{\text {ét }}\left(\bar{Y}, \mathbb{Q}_{l}(-p)\right) .
$$

\section{Reduction to the strictly semi-stable local case}

3.1. It is a local problem to check Assumption 1.3. We describe how this assumption can be checked for a strictly semi-stable model. This is sufficient if our variety has potentially semi-stable reduction. Let $X$ be a strictly semi-stable $S$-scheme as in Section 2. Instead of Assumption 1.3, we consider:

3.2. Assumption. - For all $p \geqslant 0$, the cycle class map induces an injection

$$
\mathrm{cl}: A_{p}(Y) \longrightarrow H_{2 p}^{\text {ét }}\left(\bar{Y}, \mathbb{Q}_{l}(-p)\right)
$$

$4^{\mathrm{e}}$ SÉRIE - TOME $34-2001-\mathrm{N}^{\circ} 4$ 
and the complex

$$
A_{d+1-p}(Y) \stackrel{i^{*} i_{*}}{\longrightarrow} A^{p}(Y) \stackrel{\cdot \cap[Y]}{\longrightarrow} A_{d-p}(Y)
$$

is exact.

The following result is an adaptation of Theorem 6.11 in [12] to our situation. Let $K$ be a number field with ring of integers $\mathcal{O}_{K}, S=\operatorname{Spec} \mathcal{O}_{K}$ and $\eta=\operatorname{Spec} K$. For a prime $\mathfrak{p}$ of $\mathcal{O}_{K}$, we denote by $S_{\mathfrak{p}}$ the spectrum of the completion of the localization of $\mathcal{O}_{K}$ at $\mathfrak{p}$. Let $X_{\eta}$ be a smooth projective $\eta$-variety. A model $X$ of $X_{\eta}$ over $S$ has bad reduction at a prime $\mathfrak{p}$ if $X_{\mathfrak{p}}=X \times_{S} S_{\mathfrak{p}}$ is not smooth over $S_{\mathfrak{p}}$.

3.3. Proposition. - Let $X_{\eta}$ be a d-dimensional smooth projective $K$-variety. Suppose that $X_{\eta}$ has a regular model $X$ which is flat and projective over $S$. For each place $\mathfrak{p}$ of bad reduction of $X$, we assume that there exists a finite flat morphism $S_{\mathfrak{p}}^{\prime} \rightarrow S_{\mathfrak{p}}$ of spectra of complete discrete valuation rings such that the following holds. The base change $X_{\mathfrak{p}}^{\prime}$ of $X_{\mathfrak{p}}$ to $S_{\mathfrak{p}}^{\prime}$ admits a projective $S_{\mathfrak{p}}^{\prime}$-morphism $X_{\mathfrak{p}}^{\prime \prime} \rightarrow X_{\mathfrak{p}}^{\prime}$ which induces an isomorphism of generic fibers such that $X_{\mathfrak{p}}^{\prime \prime}$ is strictly semi-stable over $S_{\mathfrak{p}}^{\prime}$ and satisfies Assumption 3.2. Then the map

$$
\varphi_{X}: C H_{\mathrm{fin}}^{d+1-p}(X)_{\mathbb{Q}}^{\perp} \longrightarrow C H^{p}\left(X_{\eta}\right)_{\mathbb{Q}}^{0}
$$

is surjective for all $p \geqslant 0$.

Proof. - Let $\alpha_{\eta}$ be a class in $C H^{p}\left(X_{\eta}\right)_{\mathbb{Q}}^{0}$. We fix an extension $\alpha$ of $\alpha_{\eta}$ in the group $C H^{p}(X)_{\mathbb{Q}}^{0}$. Let $\mathfrak{p}$ be a prime of bad reduction. We have natural maps $j_{\mathfrak{p}}: X_{\mathfrak{p}} \rightarrow X$ and $g_{\mathfrak{p}}: X_{\mathfrak{p}}^{\prime \prime} \rightarrow X_{\mathfrak{p}}$. The morphism $g_{\mathfrak{p}}$ is a projective factorable 1.c.i. morphism of relative dimension zero. The restriction of $g_{\mathfrak{p}}$ to generic fibers is finite. Therefore we may assume without loss of generality that $\alpha$ was chosen such that there exists an element $\alpha_{\mathfrak{p}}$ in $C H^{p}\left(X_{\mathfrak{p}}^{\prime \prime}\right)_{\mathbb{Q}}^{0}$ with

$$
j_{\mathfrak{p}}^{*}(\alpha)=g_{\mathfrak{p} *}\left(\alpha_{\mathfrak{p}}\right) .
$$

Let $X_{\eta}^{\prime}$ be the generic and $i_{\mathfrak{p}}^{\prime \prime}: Y^{\prime \prime} \rightarrow X_{\mathfrak{p}}^{\prime \prime}$ the special fiber of $X_{\mathfrak{p}}^{\prime \prime}$ over $S_{\mathfrak{p}}^{\prime}$. The specialization map

$$
\operatorname{sp}_{C H}: C H_{d-p}\left(X_{\eta}^{\prime}\right) \longrightarrow C H_{d-p}\left(Y_{\mathfrak{p}}^{\prime \prime}\right)
$$

maps $\left.\alpha_{\mathfrak{p}}\right|_{X_{\eta}^{\prime}}$ to $\left(i_{\mathfrak{p}}^{\prime \prime}\right)^{*}\left(\alpha_{\mathfrak{p}}\right) \cap\left[Y_{\mathfrak{p}}^{\prime \prime}\right]$. It follows from the compatibility of the cycle class map with specialization $[12,5.8]$ that $\left(i_{\mathfrak{p}}^{\prime \prime}\right)^{*} \alpha_{\mathfrak{p}} \cap\left[Y_{\mathfrak{p}}^{\prime \prime}\right]$ is homologically trivial. We conclude from (26) that the class of $\left(i_{\mathfrak{p}}^{\prime \prime}\right)^{*} \alpha_{\mathfrak{p}}$ in $A^{p}\left(Y_{\mathfrak{p}}^{\prime \prime}\right)$ lies in the kernel of the right-hand map in (27). It follows that there exists an element $\alpha_{\mathfrak{p}}^{\prime} \in C H_{d+1-p}\left(Y_{\mathfrak{p}}^{\prime \prime}\right)$ such that

$$
\left(i_{\mathfrak{p}}^{\prime \prime}\right)^{*} \alpha_{\mathfrak{p}}=\left(i_{\mathfrak{p}}^{\prime \prime}\right)^{*}\left(i_{\mathfrak{p}}^{\prime \prime}\right)_{*} \alpha_{\mathfrak{p}}^{\prime}
$$

holds in $A^{p}\left(Y_{\mathfrak{p}}^{\prime \prime}\right)$. By modifying $\alpha$ in closed fibers over bad reduction places, we may find a global class $\alpha^{0} \in C H^{p}(X)_{\mathbb{Q}}^{0}$ which is an extension of $\alpha_{\eta}$ to $X$ and satisfies

$$
j_{\mathfrak{p}}^{*}\left(\alpha^{0}\right)=j_{\mathfrak{p}}^{*}(\alpha)-\left(g_{\mathfrak{p}}\right)_{*}\left(i_{\mathfrak{p}}^{\prime \prime}\right)_{*}\left(\alpha_{\mathfrak{p}}^{\prime}\right)
$$

for all primes $\mathfrak{p}$ of bad reduction. We claim that $\alpha^{0}$ is contained in $C H_{\text {fin }}^{d+1-p}(X)_{\mathbb{Q}}^{\perp}$. We fix a prime $\mathfrak{p}$ of $\mathcal{O}_{K}$ and an element $\beta$ in the Chow group $C H_{p}\left(Y_{\mathfrak{p}}\right)_{\mathbb{Q}}$ of the special fiber $i_{\mathfrak{p}}: Y_{\mathfrak{p}} \rightarrow X_{\mathfrak{p}}$ of $X$ at $\mathfrak{p}$. We have to show that $<\alpha^{0},\left(i_{\mathfrak{p}}\right)_{*}(\beta)>_{X}$ vanishes. The good reduction case is already 
discussed in [12, proof of 6.11]. Suppose that $X$ has bad reduction at $\mathfrak{p}$. We have intersection pairings with supports on the regular schemes $X_{\mathfrak{p}}$ and $X_{\mathfrak{p}}^{\prime \prime}[9,8.3],[12,5.3]$ :

$$
\begin{gathered}
\langle., .\rangle_{X_{\mathfrak{p}}}: C H^{p}\left(X_{\mathfrak{p}}\right) \times C H_{Y_{\mathfrak{p}}}^{d+1-p}\left(X_{\mathfrak{p}}\right) \longrightarrow C H_{Y_{\mathfrak{p}}}^{d+1}\left(X_{\mathfrak{p}}\right)_{\mathbb{Q}} \stackrel{\operatorname{deg}_{Y_{\mathfrak{p}}}}{\longrightarrow} \mathbb{Q}, \\
\langle., .\rangle_{X_{\mathfrak{p}}^{\prime \prime}}: C H^{p}\left(X_{\mathfrak{p}}^{\prime \prime}\right) \times C H_{Y_{\mathfrak{p}}^{\prime \prime}}^{d+1-p}\left(X_{\mathfrak{p}}^{\prime \prime}\right) \longrightarrow C H_{Y_{\mathfrak{p}}^{\prime \prime}}^{d+1}\left(X_{\mathfrak{p}}^{\prime \prime}\right)_{\mathbb{Q}} \stackrel{\operatorname{deg}_{Y_{\mathfrak{p}}^{\prime \prime}}^{\longrightarrow}}{\longrightarrow} .
\end{gathered}
$$

It is shown in the proof of part 7) of Theorem 4.4.3 in [10] that they satisfy the projection formula

$$
\left\langle g_{\mathfrak{p} *} \alpha, \beta\right\rangle_{X_{\mathfrak{p}}}=\left\langle\alpha, g_{\mathfrak{p}}^{*} \beta\right\rangle_{X_{\mathfrak{p}}^{\prime \prime}}
$$

for $\alpha \in C H^{p}\left(X_{\mathfrak{p}}^{\prime \prime}\right)$ and $\beta \in C H_{Y_{\mathfrak{p}}}^{d+1-p}\left(X_{\mathfrak{p}}\right)$. The map $g_{\mathfrak{p}}^{*}$ is defined in loc. cit. 4.4 .1 and maps $C H_{Y_{\mathfrak{p}}}^{p}\left(X_{\mathfrak{p}}\right)$ to $C H_{Y_{\mathfrak{p}}^{\prime \prime}}^{p}\left(X_{\mathfrak{p}}^{\prime \prime}\right)$. The pairing (28) describes the local contribution to the pairing (6) at the prime $\mathfrak{p}$. Given $\beta \in C H_{p}\left(Y_{\mathfrak{p}}\right)$, we have

$$
\begin{aligned}
\left\langle\alpha^{0}, i_{\mathfrak{p} *} \beta\right\rangle_{X} & =\left\langle j_{\mathfrak{p}}^{*} \alpha^{0}, i_{\mathfrak{p} *} \beta\right\rangle_{X_{\mathfrak{p}}} \\
& =\left\langle g_{\mathfrak{p} *}\left(\alpha_{\mathfrak{p}}-\left(i_{\mathfrak{p}}^{\prime \prime}\right)_{*} \alpha_{\mathfrak{p}}^{\prime}\right), i_{\mathfrak{p} *} \beta\right\rangle_{X_{\mathfrak{p}}} \\
& =\left\langle\alpha_{\mathfrak{p}}-\left(i_{\mathfrak{p}}^{\prime \prime}\right)_{*} \alpha_{\mathfrak{p}}^{\prime}, g_{\mathfrak{p}}^{*} i_{\mathfrak{p} *} \beta\right\rangle_{X_{\mathfrak{p}}^{\prime \prime}}
\end{aligned}
$$

The element $g_{\mathfrak{p}}^{*} i_{\mathfrak{p} *} \beta$ in $C H_{Y_{\mathfrak{p}}^{\prime \prime}}^{d+1-p}\left(X_{\mathfrak{p}}^{\prime \prime}\right)_{\mathbb{Q}}=C H_{p}\left(Y_{\mathfrak{p}}^{\prime \prime}\right)_{\mathbb{Q}}$ induces an element in $A_{p}\left(Y_{\mathfrak{p}}^{\prime \prime}\right)$. The pairing (29) can be described as [12, 5.3]

$$
C H^{p}\left(X_{\mathfrak{p}}^{\prime \prime}\right) \times C H_{p}\left(Y_{\mathfrak{p}}^{\prime \prime}\right) \longrightarrow \mathbb{Q},(\alpha, \beta) \mapsto \operatorname{deg}_{Y^{\prime \prime}}\left(\left(i_{\mathfrak{p}}^{\prime \prime}\right)^{*} \alpha \cap \beta\right)
$$

where.$\cap$. refers to the cap product pairing (14). We recall that (14) factors through the groups in 2.6. We obtain

$$
\left\langle\alpha^{0}, i_{\mathfrak{p} *} \beta\right\rangle_{X}=\operatorname{deg}_{Y_{\mathfrak{p}}^{\prime \prime}}\left(\left(i_{\mathfrak{p}}^{\prime \prime}\right)^{*}\left(\alpha_{\mathfrak{p}}-\left(i_{\mathfrak{p}}^{\prime \prime}\right)_{*} \alpha_{\mathfrak{p}}^{\prime}\right) \cap\left(g_{\mathfrak{p}}^{*} i_{\mathfrak{p} *} \beta\right)\right)=0
$$

as $\left(i_{\mathfrak{p}}^{\prime \prime}\right)^{*}\left(\alpha_{\mathfrak{p}}-\left(i_{\mathfrak{p}}^{\prime \prime}\right)_{*} \alpha_{\mathfrak{p}}^{\prime}\right)$ vanishes in $A^{p}\left(Y_{\mathfrak{p}}^{\prime \prime}\right)$.

\section{Toric varieties and toric fibrations}

4.1. All varieties in this section are defined over a finite field $k$. For a smooth projective variety $X$, we denote by $A^{p}(X)$ the image of $C H^{p}(X)_{\mathbb{Q}}$ under the cycle class map into $H_{\text {ét }}^{2 p}\left(\bar{X}, \mathbb{Q}_{l}(p)\right)$.

4.2. We recall some useful properties of toric varieties. For any toric variety $Z$ for some split torus $T$ and any variety $Y$, the natural map

$$
C H .(Y) \otimes_{\mathbb{Z}} C H .(Z) \longrightarrow C H .(Y \times Z)
$$

is an isomorphism [6, Theorem 2]. If the toric variety $Z$ is smooth and proper, the cycle class map induces an isomorphism (loc. cit. Corollary to Theorem 2)

$$
\operatorname{cl} \otimes_{\mathbb{Z}} \mathbb{Q}_{l}: C H^{p}(Z)_{\mathbb{Q}_{l}} \stackrel{\sim}{\longrightarrow} H_{\text {ét }}^{2 p}\left(\bar{Z}, \mathbb{Q}_{l}(p)\right) .
$$


The Chow group $C H$. $(Z)$ is a free $\mathbb{Z}$-module which has a basis given by classes of closures of $T$-orbits in $Z[8,5.2]$.

4.3. Let $G$ be a split semi-abelian scheme. The group scheme $G$ is an extension of an abelian variety $A$ by a torus $T$ which is split over $k$. Let $T \hookrightarrow Z$ be a smooth projective toric variety. We are interested in the contraction product $P=G \times{ }^{T} Z$. We recall some facts about algebraic cycles on the toric fibration $P$ over $A$ from Section 2 in [14]. We have a cartesian square

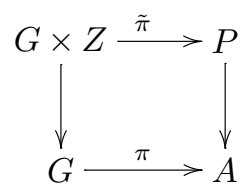

where $\pi$ and $\tilde{\pi}$ describe products of $\mathbf{G}_{m}$-torsors which are algebraically equivalent to zero. This yields isomorphisms [14, Lemma 9]

$$
C H^{\cdot}(A)_{\mathbb{Q}} \otimes_{\mathbb{Q}} C H^{\cdot}(Z)_{\mathbb{Q}} \stackrel{\sim}{\longrightarrow} C H^{\cdot}(A \times Z)_{\mathbb{Q}} \stackrel{\left(\pi \times \mathrm{id}_{z}\right)^{*}}{\longrightarrow} C H^{\cdot}(G \times Z)_{\mathbb{Q}} \stackrel{\tilde{\pi}^{*}}{\sim} C H^{\cdot}(P)_{\mathbb{Q}} .
$$

Their composition defines an isomorphism of $\mathbb{Q}$-algebras

$$
\varphi_{C H}: C H^{\cdot}(A)_{\mathbb{Q}} \otimes_{\mathbb{Q}} C H^{\cdot}(Z)_{\mathbb{Q}} \stackrel{\sim}{\longrightarrow} C H^{\cdot}(P)_{\mathbb{Q}} .
$$

The map $\varphi_{C H}$ is $\mathrm{CH}^{\cdot}(A)$-linear and has the following description (loc. cit. Lemma 10). Given an element $\alpha$ in $C H^{p}(A)_{\mathbb{Q}}$ and a $T$-stable subvariety $V$ of $Z$, we have

$$
\varphi_{C H}(\alpha \otimes[V])=\pi^{*}(\alpha) \cdot\left[G \times{ }^{T} V\right] .
$$

There is a corresponding map $\varphi_{H}$ in $l$-adic cohomology for which the diagram

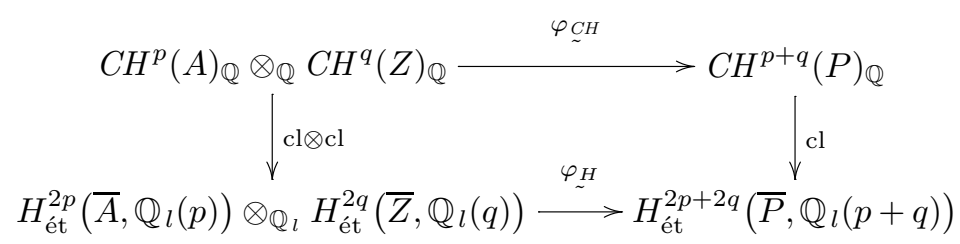

commutes. The map $\varphi_{H}$ is an $H_{\text {ét }}\left(\bar{A}, \mathbb{Q}_{l}(\cdot)\right)$-linear isomorphism of $\mathbb{Q}_{l}$-algebras.

4.4. LEMMA. - For a smooth projective toric fibration $P$ as above, the map (31) induces an isomorphism

$$
\varphi_{A}: A^{\cdot}(A) \otimes_{\mathbb{Q}} A^{\cdot}(Z) \stackrel{\sim}{\longrightarrow} A^{\cdot}(P) .
$$

Proof. - Observe that the tensor product of Chow groups is taken over $\mathbb{Q}$ whereas the tensor product of cohomology groups is taken over $\mathbb{Q}_{l}$. Our claim follows from the commutativity of (33) as (30) is an isomorphism.

4.5. Let $Z^{\prime}$ be a second smooth projective toric variety for a split torus $T^{\prime}$. We assume that there is a surjection $t: T \rightarrow T^{\prime}$ and a closed immersion $j: Z^{\prime} \rightarrow Z$ which identifies $Z^{\prime}$ with 
the closure of a $T$-orbit in $Z$ and is compatible with torus actions under $t$. Let $G^{\prime}$ be the push-out of the extension $G$ with respect to $t$ and $\mathrm{id}_{A}$. We set $P^{\prime}=G^{\prime} \times{ }^{T^{\prime}} Z^{\prime}=G \times^{T} Z^{\prime}$ and denote by $f: P^{\prime} \hookrightarrow P$ the natural map. Let $e$ denote the difference $\operatorname{dim}(P)-\operatorname{dim}\left(P^{\prime}\right)=$ $\operatorname{dim}(Z)-\operatorname{dim}\left(Z^{\prime}\right)$.

4.6. LEMMA. - The diagrams

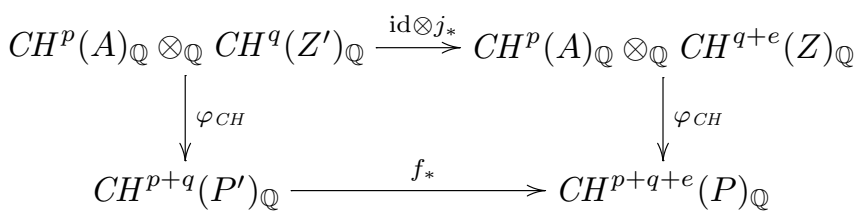

and

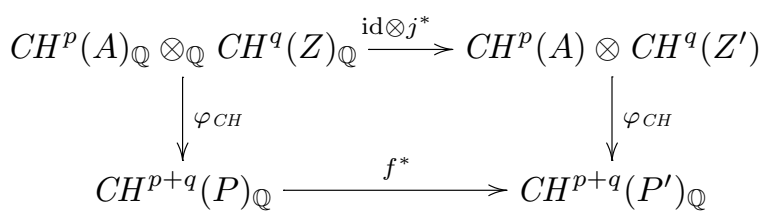

as well as the corresponding diagrams in l-adic cohomology commute.

Proof. - As $\varphi_{C H}$ and $\varphi_{H}$ are $C H^{\cdot}(A)_{\mathbb{Q}}$ (resp. $H_{\text {ét }}\left(\bar{A}, \mathbb{Q}_{l}(\cdot)\right)$ ) linear, it is sufficient to show (34) and (35) for elements of the form $1 \otimes \alpha$ for $\alpha \in C H^{\cdot}\left(Z^{\left({ }^{\prime}\right)}\right)$ (resp. for $\alpha \in$ $\left.H_{\text {ét }}\left(\overline{Z^{(\prime)}}, \mathbb{Q}_{l}(\cdot)\right)\right)$. We consider first the case of Chow groups. The commutativity in (35) follows from the fact that $\varphi_{C H}$ was defined in 4.3 as a composition of maps which are compatible with pull-backs. The commutativity of (34) follows easily from the description of $\varphi_{C H}$ in (32). Next we consider cohomology. It is sufficient to consider elements of the form $1 \otimes \alpha$. We use the surjectivity of (30) together with the properties of $\varphi_{H}$ described above to derive from our result for Chow groups that the corresponding diagrams in $l$-adic cohomology commute.

\section{Projective regular models for abelian varieties}

5.1. Let $K$ be a number field, $\mathcal{O}_{K}$ its ring of integers, and $A_{\eta}$ an abelian variety with semiabelian reduction over $\eta=\operatorname{Spec} K$. Using results of Chai, Faltings and Mumford, we have shown in [12] that $A_{\eta}$ admits a projective regular model over $S=\operatorname{Spec} \mathcal{O}_{K}$ which has potentially semistable reduction as required in Proposition 3.3. We recall the main existence statements from [12] and describe the models under consideration in detail. This will allow us to show in the next section that they fulfill the requirements made in Assumption 3.2. We also use the opportunity to correct the statement of [12, Theorem 4.6].

5.2. THEOREM. - Let $A_{\eta}$ be an abelian variety over $\eta$ with semi-abelian reduction. There exists a regular model $P$ of $A_{\eta}$ which is flat and projective over $S$.

Proof. - [12, Theorem 4.2].

The projective regular model $P$ in Theorem 5.2 is first constructed over the completions of the local rings of $\mathcal{O}_{K}$. A descent argument shows that these local models are already defined over the local rings of $\mathcal{O}_{K}$. The global model $P$ is obtained from gluing these local models. The construction of a projective regular model over a complete discrete valuation ring is based on Mumford's construction of degenerating families of abelian varieties. In its general form, this 
construction is due to Chai and Faltings. It describes degenerating families of abelian varieties in terms of so called degeneration data. We recall the main facts from [5, Ch. II, III], [12, Section 2].

5.3. Let $S$ be the spectrum of a complete discrete valuation ring $R$. Let $G$ be a semi-abelian $S$-scheme whose generic fiber $G_{\eta}$ is an abelian variety. Let $\mathcal{L}$ be a cubical ample invertible sheaf on $G$. Associated with $G$ is the Raynaud extension $\widetilde{G}$ [5, II.1]. The semi-abelian $S$-scheme $\widetilde{G}$ is an extension

$$
0 \longrightarrow T \longrightarrow \widetilde{G} \stackrel{\pi}{\longrightarrow} A \longrightarrow 0
$$

of an abelian $S$-scheme $A$ by a torus $T$. The invertible sheaf $\mathcal{L}$ induces a cubical ample invertible sheaf $\widetilde{\mathcal{L}}$ on $\widetilde{G}$ which admits a $T$-linearization if the torus $T$ is split over $S$. A triple $(G, \mathcal{L}, \mathcal{M})$ for $G$ and $\mathcal{L}$ as above is called a split ample degeneration over $S$ if $T$ is split and $\mathcal{M}$ is an ample invertible sheaf on $A$ such that $\pi^{*} \mathcal{M} \cong \widetilde{\mathcal{L}}$. Split ample degenerations form a category $\mathrm{DEG}_{\text {ample }}^{\text {split }}$ which is equivalent to the category $\mathrm{DD}_{\text {ample }}^{\text {split }}$ of split ample degeneration data. The category $\mathrm{DD}_{\text {ample }}^{\text {split }}$ is defined as follows ([5, III.2], [12, 2.2]). We consider data

$$
\left(A, X, Y, \phi, c, c^{t}, \tau, \mathcal{M}, \lambda_{A}, \psi, a, b\right)
$$

which fulfill the following requirements.

(d1) $A$ is an abelian $S$-scheme.

(d2) $X$ and $Y$ are free abelian groups of finite rank $r$.

(d3) $\phi: X \rightarrow Y$ is an injective homomorphism.

(d4) $c: X \rightarrow A^{t}(S)$ and $c^{t}: Y \rightarrow A(S)$ are homomorphisms of abelian groups.

(d5) $\tau$ is a trivialization

$$
\tau: \mathbf{1}_{(Y \times X)_{\eta}} \stackrel{\sim}{\longrightarrow}\left(c^{t} \times c\right)^{*} \mathcal{P}_{A, \eta}^{-1}
$$

of the $\mathbf{G}_{m}$-biextension $\left(c^{t} \times c\right)^{*} \mathcal{P}_{A, \eta}^{-1}$ over the constant $\eta$-group scheme $(Y \times X)_{\eta}$ where $\mathcal{P}_{A}$ is the Poincaré biextension over $A \times{ }_{S} A^{t}$.

(d6) $\mathcal{M}$ is a cubical ample invertible sheaf on $A$ such that the associated polarization $\lambda_{A}: A \rightarrow A^{t}$ satisfies $\lambda_{A} \circ c^{t}=c \circ \phi$.

(d7) $\psi$ is a trivialization

$$
\psi: \mathcal{O}_{Y_{\eta}} \stackrel{\sim}{\longrightarrow}\left(c^{t}\right)^{*} \mathcal{M}_{\eta}^{-1}
$$

of the cubical invertible sheaf $\left(c^{t}\right)^{*} \mathcal{M}_{\eta}^{-1}$ over the constant $\eta$-group scheme $Y_{\eta}$ which is compatible with $\tau$ in the sense that we have (compare [12,2.2])

$$
\Lambda(\psi)=\tau \circ\left(\operatorname{id}_{Y} \times \phi\right) .
$$

The functions $a: Y \rightarrow \mathbb{Z}$ and $b: Y \times X \rightarrow \mathbb{Z}$ measure the denominators which are needed to define the trivializations $\tau$ and $\psi$. Namely, $\psi$ and $\tau$ identify $c^{t}(y)^{*} \mathcal{M}$ and $\left(c^{t}(y), c(x)\right)^{*} \mathcal{P}_{A}$ with the fractional ideals $m_{R}^{a(y)}$ and $m_{R}^{b(y, x)}$ in $R$. The function $b$ is bilinear, the function $a$ satisfies $a(0)=0$, and (38) implies

$$
a\left(y+y^{\prime}\right)-a(y)-a\left(y^{\prime}\right)=b\left(y, \phi\left(y^{\prime}\right)\right) \quad \forall y, y^{\prime} \in Y .
$$

The positivity condition requires that Eq. (39) define a positive definite bilinear form. Data (37) as above which satisfy the positivity condition form by definition the objects of the category $\mathrm{DD}_{\text {ample }}^{\text {split }}$ of split ample degeneration data. 
5.4. We recall the definition of admissible cone decompositions from [12, 3.1]. We consider data $(X, Y, \phi, a, b)$ as in (37). Hence $X$ and $Y$ are free abelian groups of finite rank $r, \phi: X \rightarrow Y$ is an injective homomorphism, $b: Y \times X \rightarrow \mathbb{Z}$ is a bilinear function such that the induced form $b(., \phi()$.$) is positive definite, and a: Y \rightarrow \mathbb{Z}$ is a function which satisfies $a(0)=0$ and (39). There is a natural action of $Y$ on $\tilde{X}^{*}=X^{*} \times \mathbb{Z}$ via

$$
S_{y}(l, s)=(l+s b(y, .), s)
$$

for $y \in Y, l \in X^{*}=\operatorname{Hom}_{\mathbb{Z}}(X, \mathbb{Z})$, and $s \in \mathbb{Z}$. Let $\left\{\sigma_{\alpha}\right\}_{\alpha \in I}$ be a (not necessarily finite) rational polyhedral cone decomposition of the $Y$-stable cone

$$
\mathcal{C}=\left(X_{\mathbb{R}}^{*} \times \mathbb{R}_{>0}\right) \cup\{0\}
$$

in $\tilde{X}_{\mathbb{R}}^{*}$. The cone decomposition $\left\{\sigma_{\alpha}\right\}_{\alpha \in I}$ is $Y$-admissible if the collection of the cones $\sigma_{\alpha}$ is stable under the action of $Y$ and if there are only finitely many orbits. We call a cone decomposition $\left\{\sigma_{\alpha}\right\}_{\alpha \in I}$ of $\mathcal{C}$ semi-stable if $\left\{\sigma_{\alpha}\right\}_{\alpha \in I}$ is smooth $[12,1.11]$ and all onedimensional cones have a generator in $X^{*} \times\{1\}$. Given a $Y$-admissible cone decomposition $\left\{\sigma_{\alpha}\right\}_{\alpha \in I}$, we define the notion of a $k$-twisted $Y$-admissible polarization function $\varphi$ for $\left\{\sigma_{\alpha}\right\}_{\alpha \in I}$ as in $[12,3.1]$.

Let $\left\{\sigma_{\alpha}\right\}_{\alpha \in I}$ be a smooth $Y$-admissible cone decomposition of $\mathcal{C}$. Let $I^{+}$be the set $I$ with the index of the zero cone removed. The group $Y$ acts on $I$ and $I^{+}$. We denote by $I_{Y}$ and $I_{Y}^{+}$ the orbit spaces for this action. Observe that $I_{Y}$ and $I_{Y}^{+}$are finite sets. Let $\alpha$ be an index in $I^{+}$, i.e., $\sigma_{\alpha} \neq\{0\}$. Let $\tilde{X}\left(\sigma_{\alpha}\right)$ be the subgroup of $\tilde{X}=X \times \mathbb{Z}$ given by all elements which are perpendicular to $\tilde{X}^{*} \cap \sigma_{\alpha}$ with respect to the canonical pairing between $\tilde{X}$ and $\tilde{X}^{*}$. The projection from $\tilde{X}$ to $X$ induces an injection of the free abelian group $\tilde{X}\left(\sigma_{\alpha}\right)$ into $X$. Associated with each $\alpha \in I^{+}$is a cone decomposition $\widetilde{\operatorname{star}}\left(\sigma_{\alpha}\right)$ which is given as follows. Let $\operatorname{star}\left(\sigma_{\alpha}\right)$ be the star of $\sigma_{\alpha}$ which is the collection of all cones $\sigma_{\beta}$ in $\left\{\sigma_{\alpha}\right\}_{\alpha \in I}$ such that $\sigma_{\alpha}$ is a face of $\sigma_{\beta}$. Then $\widetilde{\operatorname{star}}\left(\sigma_{\alpha}\right)$ is by definition the smooth rational polyhedral cone decomposition in $\tilde{X}\left(\sigma_{\alpha}\right)_{\mathbb{R}}^{*}$ which is given by the image of $\operatorname{star}\left(\sigma_{\alpha}\right)$ under the natural projection.

5.5. We work over the spectrum $S$ of a complete discrete valuation ring $R$ and use the notations from 2.1. Let $A_{\eta}$ be an abelian variety over $\eta$ which has semi-abelian reduction and is polarized by an ample invertible sheaf $\mathcal{L}_{\eta}$. The connected component $G$ of the Néron model of $A_{\eta}$ is a semi-abelian $S$-scheme. The invertible sheaf $\mathcal{L}_{\eta}$ extends uniquely to a cubical ample invertible sheaf $\mathcal{L}$ on $G$ and induces an invertible sheaf $\widetilde{\mathcal{L}}$ on the Raynaud extension (36). If we replace $S$ by a finite, étale extension, we may assume that the torus $T$ is split and that $\widetilde{\mathcal{L}}$ descends to a cubical ample invertible sheaf $\mathcal{M}$ on $A$. The triple $(G, \mathcal{L}, \mathcal{M})$ defines a split ample degeneration over $S$ as in 5.3. We denote by

$$
\left(A, X, Y, \phi, c, c^{t}, \tau, \mathcal{M}, \lambda_{A}, \psi, a, b\right)
$$

the set of degeneration data associated with $(G, \mathcal{L}, \mathcal{M})$. We obtain in particular data $(X, Y, \phi, a, b)$ as described in 5.4. In [12, Section 3], we have constructed a projective regular compactification of $G$ from a smooth $Y$-admissible cone decomposition $\left\{\sigma_{\alpha}\right\}_{\alpha \in I}$ and from a $k$-twisted $Y$-admissible polarization function $\varphi$ for $\left\{\sigma_{\alpha}\right\}_{\alpha \in I}$. The existence of such a pair $\left(\left\{\sigma_{\alpha}\right\}_{\alpha \in I}, \varphi\right)$ was shown in loc. cit. 3.3. The compactification of $G$ constructed from $\left(\left\{\sigma_{\alpha}\right\}_{\alpha \in I}, \varphi\right)$ is a strictly semi-stable $S$-scheme if and only if the cone decomposition $\left\{\sigma_{\alpha}\right\}_{\alpha \in I}$ is semi-stable. A given $Y$-admissible cone decomposition admits a semi-stable refinement if we are allowed to change the integral structure on $X_{\mathbb{R}}^{*}$. The change of the integral structure on $X_{\mathbb{R}}^{*}$ corresponds to a ramified 
base extension of $S$. Therefore we replace $S$ by some finite flat extension which we allow this time to be ramified. After such a suitably ramified extension of $S$, we may find a refinement of a given $Y$-admissible cone decomposition with the following properties. The refinement is a semistable $Y$-admissible cone decomposition $\left\{\sigma_{\alpha}\right\}_{\alpha \in I}$ which contains the cone $\sigma_{T}=\{0\} \times \mathbb{R}_{>0}$, satisfies $S_{y}\left(\sigma_{\alpha}\right) \cap \sigma_{\alpha}=\{0\}$ for all $y \in Y-\{0\}$, and admits a $k$-twisted $Y$-admissible polarization function $\varphi$ for a suitable $k \in \mathbb{N}[12,4.5]$. For $\alpha \in I^{+}$, the injection of $\tilde{X}\left(\sigma_{\alpha}\right)$ into $X$ defined above determines a surjection of split tori over $k$

$$
T_{0} \longrightarrow T_{\alpha} \longrightarrow 0
$$

The quotient $G_{\alpha}$ of $G_{0}$ by the kernel of (40) is a semi-abelian $k$-scheme

$$
0 \longrightarrow T_{\alpha} \longrightarrow G_{\alpha} \longrightarrow A_{0} \longrightarrow 0 .
$$

5.6. THEOREM. - Associated with the semi-stable $Y$-admissible cone decomposition $\left\{\sigma_{\alpha}\right\}_{\alpha \in I}$ and the $k$-twisted polarization function $\varphi$, there is a strictly semi-stable scheme $P$ which is flat and projective over $S$, and an ample invertible sheaf $\mathcal{L}_{P}$ on $P$ such that:

(i) $G$ is an open subscheme of $P$ and $\left.\mathcal{L}_{P}\right|_{G}=\mathcal{L}^{\otimes k}$.

(ii) The action of $G$ on itself by translation extends to $P$.

(iii) The special fiber $P_{0}$ is a reduced divisor with strictly normal crossings on $P$. It has a natural stratification with strata $G_{\alpha}$ for $\alpha$ in $I_{Y}^{+}$. The strata are precisely the orbits for the action of $G_{0}$ on $P_{0}$ given in (ii). The special fiber $G_{0}$ of $G$ coincides with the stratum $G_{\sigma_{T}}$. There is a canonical isomorphism of $k$-varieties

$$
S_{y}: G_{\alpha} \stackrel{\sim}{\longrightarrow} G_{\beta}
$$

if $S_{y}(\alpha)=\beta$ for some $y \in Y$.

(iv) The closure $P_{\alpha}$ of the stratum $G_{\alpha}$ is the disjoint union of all $G_{\beta}$ such that $\sigma_{\alpha}$ is a face of $\sigma_{\beta}$. If we equip it with its induced reduced subscheme structure, it becomes isomorphic to the contraction product

$$
G_{\alpha} \times^{T_{\alpha}} Z_{\alpha}
$$

where $T_{\alpha} \hookrightarrow Z_{\alpha}$ is the smooth projective toric variety over $k$ associated with the cone decomposition $\widehat{\operatorname{star}}\left(\sigma_{\alpha}\right)$ defined above. For $\alpha \in I^{+}$, the canonical maps $S_{y}$ from $G_{\alpha}$ to $G_{S_{y}(\alpha)}$ induce isomorphisms $S_{y}$ from $P_{\alpha}$ to $P_{S_{y}(\alpha)}$ which fit into the commutative squares
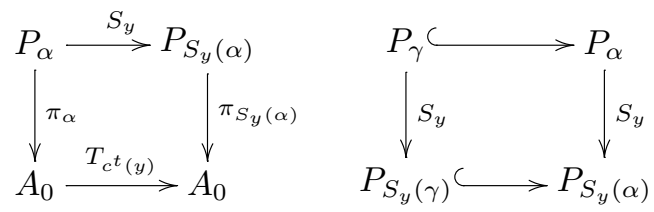

where $T_{c^{t}(y)}$ denotes translation by $c^{t}(y) \in A(S)$ and $\sigma_{\alpha}$ is a face of $\sigma_{\gamma}$.

Proof. - This follows from Theorem 3.5 and Theorem 4.7 in [12].

5.7. The construction of the models in Theorem 5.2 and Theorem 5.6 can be done in a way that is compatible with the requirements made in 3.3. In fact, we have to choose an admissible cone decomposition for each bad reduction prime in order to obtain a global model as in Theorem 5.2. 
After a suitable change of the integral structure, each of these cone decompositions admits a semi-stable refinement. The change of the integral structure corresponds locally to a finite flat ramified base change. The refinement of the cone decomposition corresponds to blowing ups in the special fiber. The combination of these modifications yields locally a strictly semi-stable model as required in Proposition 3.3.

5.8. Erratum to [12]. Let $R$ be a discrete valuation ring with quotient field $K$. Let $A_{\eta}$ be an abelian $K$-variety which has semi-abelian reduction and is polarized by an ample invertible sheaf $\mathcal{L}_{\eta}$. In [12, Theorem 4.2], we claim that there exists a discrete valuation ring $R^{\prime}$ which is a finite flat extension of $R$ such that the base change of $A_{\eta}$ to the quotient field of $R^{\prime}$ admits a projective semi-stable model over $R^{\prime}$ with properties (i)-(iv) listed in Theorem 5.6. This statement holds as stated in loc. cit. if $R$ is a complete discrete valuation ring. If $R$ is an arbitrary discrete valuation ring, the finite flat ring extension $R^{\prime}$ of $R$ constructed in loc. cit. no longer has to be a discrete valuation ring. The statement of [12, Theorem 4.2] has to be corrected as follows. In the above situation, there exists a finite field extension $K^{\prime}$ of $K$ such that the base change of $A_{\eta}$ to $K^{\prime}$ admits a projective regular model $P$ over the integral closure $R^{\prime}$ of $R$ in $K^{\prime}$. This model $P$ has semistable reduction over each localization of $R^{\prime}$ with the properties listed in [12, Theorem 4.2] and Theorem 5.6 (i)-(iv). This correction does not affect the validity of the other results in [12]. The theorem is only used in the proof of [12, Theorem 6.13] where one can work over a complete discrete valuation ring as in this paper.

\section{Reduction to the totally degenerate case}

6.1. Let $K$ be a number field and $A_{\eta}$ a $d$-dimensional abelian variety with semi-abelian reduction over $\eta=\operatorname{Spec} K$. In the last section, we have seen that $A_{\eta}$ admits a projective regular model over the ring of integers in $K$ which has potentially semi-stable reduction as required in Proposition 3.3. In this section, we show that the strictly semi-stable models constructed in Theorem 5.6 satisfy Assumption 3.2.

6.2. Let $\mathrm{R}$ be a complete discrete valuation ring with finite residue class field $k$. Let $(G, \mathcal{L}, \mathcal{M})$ be a split ample degeneration over $S$ such that the associated degeneration data

$$
\left(A, X, Y, \phi, c, c^{t}, \tau, \mathcal{M}, \lambda_{A}, \psi, a, b\right)
$$

admit a semi-stable $Y$-admissible polyhedral cone decomposition $\left\{\sigma_{\alpha}\right\}_{\alpha \in I}$ which contains the cone $\sigma_{T}=\{0\} \times \mathbb{R}_{>0}$, satisfies $S_{y}\left(\sigma_{\alpha}\right) \cap \sigma_{\alpha}=\{0\}$ for all $y \in Y-\{0\}$, and admits a $k$-twisted $Y$-admissible polarization function $\varphi$ for a suitable $k \in \mathbb{N}$. Let $P$ be the compactification of $G$ constructed in Theorem 5.6 from $\left(\left\{\sigma_{\alpha}\right\}_{\alpha \in I}, \varphi\right)$. In order to show that Assumption 3.2 holds for the model $P$, we construct a second strictly semi-stable $S$-scheme $P^{\prime}=Q$. The new scheme $Q$ is closely related to $P$ and will satisfy Assumption 3.2. Using again the pair $\left(\left\{\sigma_{\alpha}\right\}_{\alpha \in I}, \varphi\right)$, we construct $P^{\prime}=Q$ as a compactification of a totally degenerate semi-abelian $S$-scheme $G^{\prime}$ from a split ample degeneration $\left(G^{\prime}, \mathcal{L}^{\prime}, \mathcal{M}^{\prime}\right)$. We construct $\left(G^{\prime}, \mathcal{L}^{\prime}, \mathcal{M}^{\prime}\right)$ from its degeneration data

$$
\left(A^{\prime}, X^{\prime}, Y^{\prime}, \phi^{\prime}, c^{\prime},\left(c^{\prime}\right)^{t}, \tau^{\prime}, \mathcal{M}^{\prime}, \lambda_{A}^{\prime}, \psi^{\prime}, a^{\prime}, b^{\prime}\right)
$$

by Mumford's construction 5.3. We choose a prime element $\pi_{R}$ of $R$ in order to define our new degeneration data. The data (41) are defined as follows:

(d1) $A^{\prime}$ is the trivial abelian $S$-scheme.

(d2) $X^{\prime}=X$ and $Y^{\prime}=Y$.

$4^{\mathrm{e}}$ SÉRIE - TOME $34-2001-\mathrm{N}^{\circ} 4$ 
(d3) $\varphi^{\prime}=\varphi$.

(d4) $c: X^{\prime} \rightarrow A^{\prime}(S)=\{e\}$ and $c^{t}: Y^{\prime} \rightarrow\left(A^{\prime}\right)^{t}(S)=\{e\}$ are the zero maps.

(d5) The trivialization

$$
\tau^{\prime}: \mathbf{1}_{\left(X^{\prime} \times Y^{\prime}\right)_{\eta}} \stackrel{\sim}{\longrightarrow}\left(\left(c^{\prime}\right)^{t} \times c^{\prime}\right)^{*} \mathcal{P}_{A^{\prime}, \eta}^{-1}=\mathbf{1}_{\left(X^{\prime} \times Y^{\prime}\right)_{\eta}}
$$

is defined by $\tau^{\prime}\left(\mathbf{1}_{(y, x)}\right)=\pi_{R}^{-b(y, x)} \cdot \mathbf{1}_{(y, x)}$ for $y \in Y^{\prime}$ and $x \in X^{\prime}$.

(d6) $\mathcal{M}^{\prime}=\mathcal{O}_{S}$ is the structure sheaf.

(d7) We define the trivialization

$$
\psi^{\prime}: \mathcal{O}_{Y_{\eta}^{\prime}} \stackrel{\sim}{\longrightarrow}\left(c^{\prime}\right)^{t *}\left(\mathcal{M}_{\eta}^{\prime}\right)^{-1}=\mathcal{O}_{Y_{\eta}^{\prime}}
$$

by $\psi^{\prime}\left(\mathbf{1}_{y}\right)=\pi_{R}^{-a(y)} \cdot \mathbf{1}_{y}$ for all $y \in Y^{\prime}$.

It follows from the above definitions that we have

$$
\left(X^{\prime}, Y^{\prime}, \phi^{\prime}, a^{\prime}, b^{\prime}\right)=(X, Y, \phi, a, b) .
$$

We have to show that our data satisfy (38) and that the positivity condition holds. We conclude from (42) that (39) holds for our new data and that the positivity condition is satisfied. Eq. (38) is a consequence of our definition of $\tau^{\prime}$ and $\psi^{\prime}$ if one observes the compatibility (39).

The degeneration data (41) determine a split ample degeneration $\left(G^{\prime}, \mathcal{L}^{\prime}, \mathcal{M}^{\prime}\right)$ over $S$. We may use the cone decomposition $\left\{\sigma_{\alpha}\right\}_{\alpha \in I}$ and the polarization function $\varphi$ to construct a strictly semistable $S$-scheme $P^{\prime}$ with an ample invertible sheaf $\mathcal{L}_{P^{\prime}}$ which is a compactification of $G^{\prime}$ as in Theorem 5.6. We write $Q=P^{\prime}$, e for the dimension of $Q_{0}$, and denote by $i$ and $j$ the inclusions of the special fibers $P_{0}$ and $Q_{0}$ into $P$ and $Q$. As the model $Q$ is constructed from the same cone decomposition as $P$, it has a similar special fiber. The strata of the special fibers $P_{0}$ and $Q_{0}$ correspond to elements in $I_{Y}^{+}$. The $Q_{\alpha}$ for $\alpha \in I^{+}$are smooth projective toric varieties and each $P_{\alpha}$ is a contraction product $G_{\alpha} \times{ }^{T_{\alpha}} Q_{\alpha}$. We are interested in the model $Q$ as it satisfies Assumption 3.2.

6.3. THEOREM. - The cycle class in étale homology induces an isomorphism

$$
\mathrm{cl} \otimes_{\mathbb{Q}} \mathbb{Q}_{l}: A_{p}\left(Q_{0}\right)_{\mathbb{Q}_{l}} \stackrel{\sim}{\longrightarrow} \operatorname{Gr}_{0}^{W} H_{2 p}^{\text {ét }}\left(\overline{Q_{0}}, \mathbb{Q}_{l}(-p)\right) \subseteq H_{2 p}^{\text {ét }}\left(\overline{Q_{0}}, \mathbb{Q}_{l}(-p)\right)
$$

and the complex

$$
A_{e+1-p}\left(Q_{0}\right) \stackrel{j^{*} j_{*}}{\longrightarrow} A^{p}\left(Q_{0}\right) \stackrel{\cdot \cap\left[Q_{0}\right]}{\longrightarrow} A_{e-p}\left(Q_{0}\right)
$$

is exact.

Proof. - The first isomorphism follows from the bijectivity of (30) as $A_{p}\left(Q_{0}\right)$ is the cokernel of $\gamma$ in (21) and $\mathrm{Gr}_{0}^{W} H_{2 p}^{\text {ét }}\left(\overline{Q_{0}}, \mathbb{Q}_{l}(-p)\right)$ is the cokernel of $\gamma$ in (24). The exactness of (44) follows from 2.8 (compare [12, proof of 6.13]).

According to Lemma 4.4, there is for each $\alpha \in I_{Y}^{+}$a canonical isomorphism

$$
\varphi_{A}: A^{\cdot}\left(A_{0}\right) \times_{\mathbb{Q}} A^{\cdot}\left(Q_{\alpha}\right) \stackrel{\sim}{\longrightarrow} A^{\cdot}\left(P_{\alpha}\right) .
$$

The following proposition shows that this isomorphism behaves naturally with respect to the action of $Y$. 
6.4. Proposition. - Let $\alpha$ and $\beta$ be elements in $I^{+}$such that $S_{y}(\alpha)=\beta$ holds for some $y \in Y$. In this case, there are natural isomorphisms $S_{y}: Q_{\alpha} \rightarrow Q_{\beta}$ and $S_{y}: P_{\alpha} \rightarrow P_{\beta}$ and the diagram

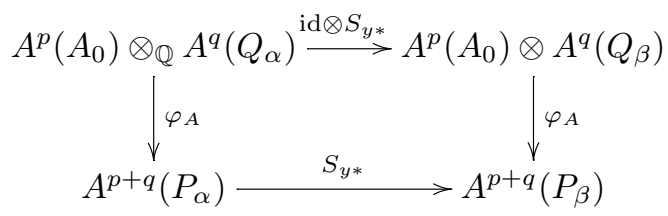

commutes.

Proof. - The maps $S_{y}$ in the diagram have been introduced in Theorem 5.6(iv). The isomorphism $S_{y}$ from $P_{\alpha}$ to $P_{\beta}$ fits into the left commutative square in loc. cit. The graph of the translation map $T_{c^{t}(y)}$ is algebraically equivalent to the graph of the identity. Hence $T_{c^{t}}(y)$ acts trivially on $A^{\cdot}\left(A_{0}\right)$ and we obtain

$$
\pi_{\alpha}^{*}(x)=\pi_{\alpha}^{*} T_{c^{t}(y)}^{*}(x)=S_{y}^{*} \pi_{\beta}^{*}(x) \quad \forall x \in A^{\cdot}\left(A_{0}\right) .
$$

The group $A^{p}\left(A_{0}\right) \otimes_{\mathbb{Q}} A^{q}\left(Q_{\alpha}\right)$ is generated by elements $x \otimes\left[Q_{\gamma}\right]$ where $x \in A^{p}\left(A_{0}\right), \gamma \in I^{+}$ such that $\sigma_{\alpha}$ is a face of $\sigma_{\gamma}$, and $\left[Q_{\gamma}\right]$ is the class of the subvariety which is given as the closure of the stratum $G_{\gamma}^{\prime}=T_{\gamma}$ corresponding to $\gamma$ in 5.6 (iii). We define $\delta \in I^{+}$by $S_{y}\left(\sigma_{\gamma}\right)=\sigma_{\delta}$. The map $S_{y}$ in the proposition maps $Q_{\gamma}$ to $Q_{\delta}$ and $P_{\gamma}$ to $P_{\delta}$. We have

$$
\begin{aligned}
S_{y *} \varphi_{A}\left(x \otimes\left[Q_{\gamma}\right]\right) & =S_{y *}\left(\pi_{\alpha}^{*} x \cdot\left[G_{\alpha} \times{ }^{T_{\alpha}} Q_{\gamma}\right]\right) \\
& =S_{y *}\left(S_{y}^{*} \pi_{\beta}^{*} x \cdot\left[P_{\gamma}\right]\right) \\
& =\pi_{\beta}^{*}(x) \cdot S_{y *}\left[P_{\gamma}\right] \\
& =\pi_{\beta}^{*}(x) \cdot\left[G_{\beta} \times{ }^{T_{\beta}} Q_{\delta}\right] \\
& =\varphi_{A}\left(x \otimes S_{y_{*}}\left[Q_{\gamma}\right]\right) .
\end{aligned}
$$

This proves our claim.

Next we check compatibility of (45) with $\gamma$ and $\rho$. We choose an ordering of the classes of onedimensional cones in $I_{Y}^{+}$, i.e. an identification of the irreducible components of $Q_{0}$ and $P_{0}$ with $\{1, \ldots, t\}$. We consider the maps $\gamma$ and $\rho$ from (21). We claim with the corresponding notation $P_{I}, Q_{I}, P^{(i)}, Q^{(i)}$ as in Section 2 the following lemma.

\subsection{LEMMA. - The diagrams}

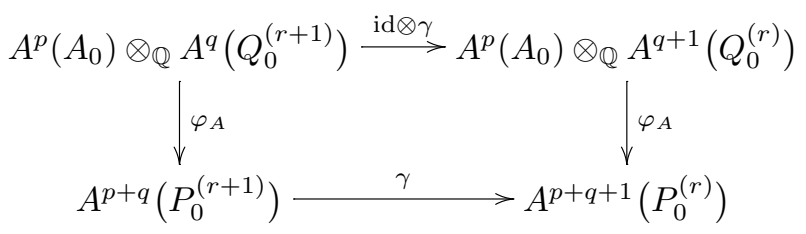


and

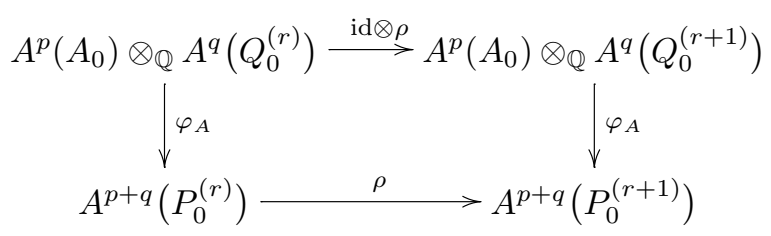

as well as the corresponding diagrams in l-adic cohomology commute.

Proof. - The maps $\gamma$ and $\rho$ are defined in terms of the maps (16) and (17). Hence it is sufficient to show the corresponding commutativity statements in the lemma for these maps. It follows from our construction and from Proposition 6.4 that we may assume therefore that we are in the situation of 4.5. Then our claim follows from Lemma 4.6.

We are ready to prove our final result.

6.6. THEOREM. - Let $P$ be a strictly semi-stable model of an abelian variety as constructed in 5.6. Then the cycle class map in étale homology induces an injection

$$
\mathrm{cl}: A_{p}\left(P_{0}\right) \longrightarrow H_{2 p}^{\text {ét }}\left(\overline{P_{0}}, \mathbb{Q}_{l}(-p)\right)
$$

and the complex

$$
A_{d+1-p}\left(P_{0}\right) \stackrel{i^{*} i_{*}}{\longrightarrow} A^{p}\left(P_{0}\right) \stackrel{\cdot \cap\left[P_{0}\right]}{\longrightarrow} A_{d-p}\left(P_{0}\right)
$$

is exact.

Proof. - We recall that $d, e$, and $g=d-e$ denote the dimensions of $P_{0}, Q_{0}$, and $A_{0}$ respectively. For all $p, q \geqslant 0$, we have a commutative diagram

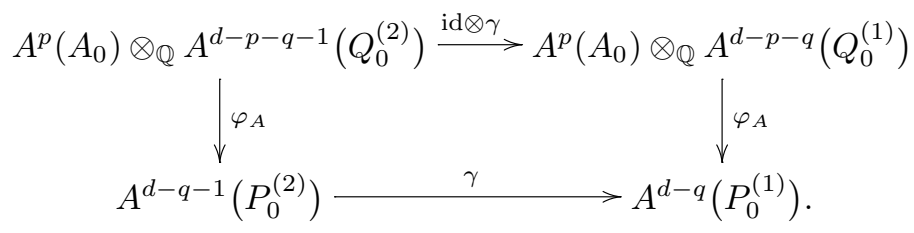

We obtain a map of the cokernels of the horizontal maps

$$
\tilde{\varphi}_{A}: A^{p}\left(A_{0}\right) \otimes_{\mathbb{Q}} A_{p+q-g}\left(Q_{0}\right) \rightarrow A_{q}\left(P_{0}\right)
$$

which induces an isomorphism

$$
\tilde{\varphi}_{A}: \bigoplus_{p} A^{p}\left(A_{0}\right) \otimes_{\mathbb{Q}} A_{p+q-g}\left(Q_{0}\right) \rightarrow A_{q}\left(P_{0}\right)
$$

The diagram (48) admits cycle class maps into the corresponding diagram in $l$-adic cohomology. We obtain a commutative diagram of cokernels

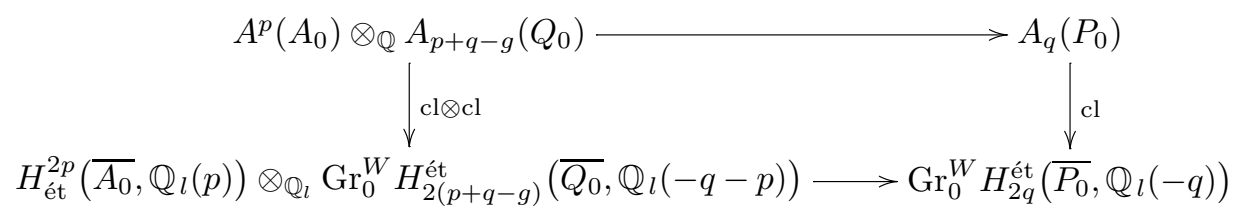


where the horizontal maps become isomorphisms if we take on the left-hand side the direct sum over all $p$. We have seen that $\operatorname{Gr}_{0}^{W} H_{2 q}^{\text {ét }}\left(\overline{P_{0}}, \mathbb{Q}_{l}(-q)\right)$ is a subspace of $H_{2 q}^{\text {ét }}\left(\overline{P_{0}}, \mathbb{Q}_{l}(-q)\right)$. It follows that (46) is injective if the right vertical map in the diagram above is injective. However this follows from the commutativity of the diagram if one observes the isomorphism (43). Next we show the equality

$$
\operatorname{ker}\left(\left.\rho\right|_{A^{p}\left(P_{0}^{(1)}\right)}\right) \cap \operatorname{Im}\left(\left.\gamma\right|_{A^{p-1}\left(P_{0}^{(2)}\right)}\right)=\operatorname{Im}\left(\left.\gamma \circ \rho\right|_{A^{p-1}\left(P_{0}^{(1)}\right)}\right)
$$

which implies the required exactness by Lemma 2.7. It follows from Lemma 6.5 that we have

$$
\begin{aligned}
\operatorname{ker}\left(\left.\rho\right|_{A^{p}\left(P_{0}^{(1)}\right)}\right) & =\bigoplus_{q^{\prime}+q=p} A^{q^{\prime}}\left(A_{0}\right) \otimes_{\mathbb{Q}} \operatorname{ker}\left(\left.\rho\right|_{A^{q}\left(Q_{0}^{(1)}\right)}\right), \\
\operatorname{Im}\left(\left.\gamma\right|_{A^{p-1}\left(P_{0}^{(2)}\right)}\right) & =\bigoplus_{q^{\prime}+q=p} A^{q^{\prime}}\left(A_{0}\right) \otimes_{\mathbb{Q}} \operatorname{Im}\left(\left.\gamma\right|_{A^{q-1}\left(Q_{0}^{(2)}\right)}\right), \\
\operatorname{Im}\left(\left.\gamma \circ \rho\right|_{A^{p-1}\left(P_{0}^{(1)}\right)}\right) & =\bigoplus_{q^{\prime}+q=p} A^{q^{\prime}}\left(A_{0}\right) \otimes_{\mathbb{Q}} \operatorname{Im}\left(\left.\gamma \circ \rho\right|_{A^{q-1}\left(Q_{0}^{(1)}\right)}\right) .
\end{aligned}
$$

We see in particular that the left-hand side of (49) equals

$$
\bigoplus_{q^{\prime}+q=p} A^{q^{\prime}}\left(A_{0}\right) \otimes_{\mathbb{Q}}\left(\left(\operatorname{ker}\left(\left.\rho\right|_{A^{q}\left(Q_{0}^{(1)}\right)}\right) \cap \operatorname{Im}\left(\left.\gamma\right|_{A^{q-1}\left(Q_{0}^{(2)}\right)}\right) .\right.\right.
$$

This proves our claim as (23) holds for $Q_{0}$ by Theorem 6.3 and Lemma 2.7.

\section{Acknowledgments}

Work on this paper started while the author was visiting the Newton institute in Cambridge. It is a pleasure to thank the institute for its hospitality, the organizers of the Arithmetic Geometry program for inviting me, and the Deutsche Forschungsgemeinschaft and the European Commission for financial support. I am also grateful to A. Abbes, A. Huber and the referees for their remarks on previous versions of this paper.

\section{REFERENCES}

[1] BeILInson A.A., Height pairings between algebraic cycles, in: Manin Yu.I. (Ed.), K-theory, Arithmetic and Geometry, Moscow 1984-86, Lect. Notes Math., Vol. 1289, Springer, Berlin, 1987, pp. 1-25 and Contemp. Math. 67 (1987) 1-24.

[2] BLOCH S., Height pairings for algebraic cycles, J. Pure Appl. Algebra 34 (1984) 119-145.

[3] Bloch S., Gillet H., Soulé C., Cycles on degenerate fibers, in: Arithmetic Geometry (Cortona, 1994), Sympos. Math. XXXVII, Cambridge Univ. Press, Cambridge, 1997, pp. 45-69.

[4] Deligne P., La conjecture de Weil II, Publ. Math. I.H.E.S. 52 (1981) 313-428.

[5] Faltings G., Chai C.-L., Degenerations of Abelian Varieties, Springer, Berlin, 1990.

[6] Fulton W., MacPherson R., Sottile F., Sturmfels B., Intersection theory on spherical varieties, J. Algebraic Geometry 4 (1995) 181-193.

[7] Fulton W., Intersection Theory, Springer, Berlin, 1984.

[8] Fulton W., Introduction to Toric Varieties, Annals of Mathematics Studies, Vol. 131, Princeton University Press, Princeton, NJ, 1993.

[9] Gillet H., SoulÉ C., Intersection theory using Adams operations, Invent. Math. 90 (1987) 243-278.

[10] Gillet H., SoulÉ C., Arithmetic intersection theory, Publ. Math. I.H.E.S. 72 (1990) 94-174.

$4^{\mathrm{e}}$ SÉRIE - TOME $34-2001-\mathrm{N}^{\circ} 4$ 
[11] Künnemann K., Higher Picard varieties and the height pairing, Amer. J. Math. 118 (1996) 781-797.

[12] KÜNNEMANN K., Projective regular models for abelian varieties, semi-stable reduction, and the height pairing, Duke Math. J. 95 (1998) 161-212.

[13] KÜnNEMAnN K., The Kähler identity for bigraded Hodge-Lefschetz modules and its application in non-archimedean Arakelov geometry, J. Algebraic Geometry 7 (1998) 651-672.

[14] KÜNnEMANn K., Algebraic cycles on toric fibrations over abelian varieties, Math. Z. 232 (1999) $427-435$.

(Manuscript received September 17, 1999;

accepted, after revision, March 27, 2000.)

\author{
Klaus KÜNNEMANN \\ NWF I-Mathematik, \\ Universität Regensburg, \\ 93040 Regensburg, Germany \\ E-mail: klaus.kuennemann@mathematik. \\ uni-regensburg.de
}

